\title{
Friction and noise suppression for force control system based on integration of observer and force sensor information
}

\author{
Yuki Nagatsu* (10) and Hideki Hashimoto
}

\begin{abstract}
Measuring and controlling external force from the environment and human beings are essential to realize the future application of the robot systems for medical, nursing, and personal support systems. This paper proposes a friction and noise suppression for force control system based on the integration of the reaction torque observer and force (torque) sensor information. The proposed method performs external force estimation by integrating the lowfrequency component information of the force sensor and the high-frequency component of the force information estimated by reaction torque observer (RTOB). By using the low-frequency component of the force sensor, the effect of static friction can be suppressed, and the high-frequency part of RTOB can be used to attenuate the noise component. As a result, the performance of the force control system can be enhanced. The proposed method is evaluated through the experiments of the force control and bilateral control as a position/force hybrid control.
\end{abstract}

Keywords: Force control, Disturbance observer, Reaction torque observer, Acceleration control, Bilateral control, Position/force hybrid control, Force sensor

\section{Introduction}

Recently, torque/force control systems used for the realization of contact motions have been widely researched [1-3]. Measuring and controlling external force from the environment and human beings are essential to realize the future application of the robot systems for medi$\mathrm{cal}$, nursing, and personal support systems. The reaction torque/force control is required for precise control, when contacting delicate objects, and to interact with human beings in open environments. In particular, robust torque/force control systems based on acceleration controllers that use disturbance observers (DOBs) $[4,5]$ have been developed as effective controllers [6,7]. Additionally, senseless torque/force control can be achieved by combining these with reaction force observers (RTOBs) [8-10]. The superiority of the observer-based force control system over the force sensor-based system is

\footnotetext{
*Correspondence: nagatsu@elect.chuo-u.ac.jp

Department of Electrical, Electronic, and Communication Engineering,

Chuo University, Kasuga Bunkyo-ku, 1-13-27, Tokyo, Japan
}

reported by [11]. Acceleration-based reaction torque control can be applied to many control systems, such as bilateral teleoperation systems [12] and human motion imitation, by using a motion-copying system [13, 14]. However, RTOB requires a modeling and compensation of friction torque to estimate the external force $[5,8,9]$. Compensation for friction torque is a general problem in force control, not limited to RTOB-based techniques [15-17]. The modeling and identification methods have been proposed in the literature [18-20]. However, the detailed and accurate modeling and identification of the friction torque, especially Coulomb friction, is challenging to achieve. Although compensation methods have been reported to reduce the influence of friction by introducing a mechanism such as a twin drive system [21] or a non-contact magnetic gear [22], it leads to an increase in size and complexity of the system. Although the utilization of a direct drive (DD) motor is conceivable to avoid friction due to the reduction gear, the output torque of the DD motor is usually smaller than that of the geared motor. The utilization of force sensors, however, 
is sensitive to noise and limit the bandwidth of the force control system by implementing a low-pass filter to suppress the noise. As described above, the RTOB-based force control has a problem due to friction. Also, the force sensor-based method has a problem in bandwidth limitation of force sensing due to noise.

Therefore, the purpose of this paper is to suppress both the friction of the geared motor and the noise of the force sensor, simultaneously. To achieve this purpose, this paper proposes a method for estimating external force by taking advantage of both RTOB and force sensor. The proposed method uses the low-frequency component of the force sensor to suppress the effect of friction and uses the high-frequency component of the estimated external force by RTOB to suppress the effect of noise of the sensor. This paper also proposes a force control system using the force estimation method integrating these two force information. The proposed force control system is also applied to bilateral control, which is a position/force hybrid control. The proposed method improves the disturbance (friction) suppression characteristics compared to the RTOB-based method and improves the noise sensitivity compared to the force sensor-based method.

\section{Related work and the proposed method}

This section describes much more detailed technical aspects with the conventional work for the external force estimation/measurement and the proposed method.

\section{Force control with force sensor}

Force sensors are used to measure the external force information. The force sensor can measure only the external force of the contacting part. A DOB-based force control system combined with a force sensor can be constructed [23, 24]. The force sensor-based force control system has a problem of deterioration of the performance due to sensor-noise [7]. If a low-pass filter is used to suppress the noise of force sensor, however, the limited force measurement bandwidth leads to deterioration of the control performance. The phase lag due to the LPF also leads to deterioration of the stability [7]. Force measurement methods combined with Kalman filter techniques have been developed to reduce the noise effect. To address reducing a noise effect, force-measurement methods combined with Kalman filter techniques have been developed [25-27]. However, Kalman filter-based methods require a precise control plant model and analysis for noise characteristics.

\section{Force control with external torque estimation by RTOB}

The RTOB have been developed to realize force sensor-less force control system. The RTOB estimate the external torque by using an actuator torque, an angular acceleration information, and an modelled (identified) friction torque $[5,8,9]$. If the resolution of the position encoder of the actuator is sufficiently high, the noiseeffect appears in the estimated force is can be neglected because the angular acceleration information is calculated from the position information. If the RTOB applied to the geared motor, friction torque due to the reduction gear should be considered. Modeling and identification of the friction torque are necessary to subtract the friction torque component from the total disturbance torque. Friction torque is usually modeled as viscous friction and Coulomb friction [8,9]. However, it is difficult to model and identify accurate friction, especially in the low-speed region. On the other hand, much more detailed friction models considering the sigmoid function and Stribeck friction have been proposed [18, 28]. Also, a method has been proposed to identify the friction torque by using an simulated annealing method [29] or an on-line least mean square error algorithm (RLMS) [9]. However, if a detailed model is constructed, the number of parameters to be identified increases, and the design procedure becomes complicated. Also, It is challenging to know the actual friction torque information, no matter how detailed the model is. The identification error of the friction directly acts as a disturbance in the force control system. For example, the identification error of the Coulomb friction acts on the system as a step disturbance as follows:

$$
\tau^{\text {coul }}-\hat{\tau}^{\text {coul }}=\Delta \tau^{\text {coul }}
$$

Here, $\tau^{\text {coul }}, \hat{\tau}^{\text {coul }}$, and $\Delta \tau^{\text {coul }}$ represent the actual Coulomb friction, the identified Coulomb friction, and the identification error of Coulomb friction, respectively, which are all constants. Further, although no external force is received, the identification error of the frictional force is erroneously detected as the external force. Also, if a human operates an actuator as in bilateral control, the identification error of the frictional force appears as an operating force. Therefore, the friction force degrades the operability (back drivability) of the system.

\section{Overview of the proposed external force estimation}

As described above, the force sensor-based force control system is affected by force-sensor noise. On the other hand, the RTOB-based force control system has difficulties in compensating the friction force. In order to address these problems, this paper proposes a novel external force estimation method utilizing the both advantages of the force sensor and RTOB. The conceptual figure of the proposed method is shown in Fig. 1. The proposed external force estimation method is composed of two elements: a low-frequency component of the force information measured by a force sensor and a 


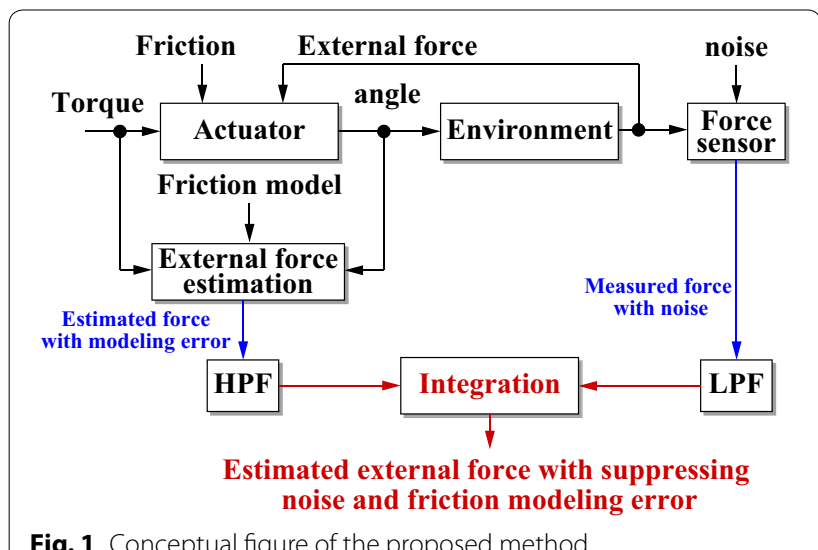

Fig. 1 Conceptual figure of the proposed method

high-frequency component of the force information estimated by RTOB. The proposed method requires only the viscous friction model and its identified value. By using the low-frequency component of the force information, the proposed method can estimate the accurate external force information with suppressing noise. Also, the proposed method can obtain the external force information in the high-frequency domain by RTOB with suppressing Coulomb friction because the Coulomb friction can be regarded as a step disturbance. As a result, the proposed method realizes friction and noise suppression simultaneously.

As an application of the proposed method, accurate and high-output force control by geared motors is considered. Also, the proposed method can be applied to the bilateral control system composed of geared actuators with high-output force and high back-drivability.

Here, the assumptions and limitations of the proposed method in this paper are described below. This paper assumes that only the friction torque and external torque are considered as the disturbance torque (force) added to actuators for the simplicity. The disturbance torque due to the modeling errors of the torque coefficient and motor inertia, and gravitational torque is assumed to be negligibly small. As a limitation of the proposed method, the resolution of the position encoder needs to be sufficiently high. This is because external force estimation requires acceleration information obtained by differentiating position information. Unless the noise level due to the RTOB is lower than the noise level of the force sensor, the effect of the proposed method will be little. However, since a high encoder resolution is required for accurate disturbance estimation as well as external force estimation, it is not the particular case that a high-resolution encoder is implemented to the proposed method. Further, by equipping a reduction gear, it is possible to improve the equivalent position-resolution of the output axis, which is suitable as an application of the proposed method.

\section{Force control system based on DOB}

This section describes force control systems based on DOB [4, 6-8]. First, an acceleration controller to realize robust control system by using DOB is explained. Next, a force control system using a disturbance observer and force sensor is described. The force control system using DOB and RTOB is also explained.

\section{Acceleration controller based on $\mathrm{DOB}$}

The motion equation of the force control system with a geared motor is represented as follows:

$$
\begin{aligned}
& J_{G} s^{2} q=\tau^{\text {ref }}-\tau^{\text {load }}=K_{t G} I^{\text {ref }}-\tau^{\text {load }} \\
& \tau^{\text {load }}=\tau^{\text {ext }}+\tau^{\text {coul }}+D_{G} \omega
\end{aligned}
$$

where $q, \omega, \tau, I, J_{G} K_{t G}, D_{G}$, superscript ref, load, ext and coul represent the angle, angular velocity, torque, current, inertia, torque constant, viscous coefficient, reference value, load torque, external torque, and Coulomb friction, respectively. The inertia, torque constant and viscous coefficient represent values converted from the motor side values $\left(J, K_{t}\right.$ and $\left.D\right)$ to the output side by using gear ratio as $J_{G}=G_{r}^{2} J, K_{t G}=G_{r} K_{t}$ and $D_{G}=G_{r}^{2} D$. The external torque is modeled below.

$$
\tau^{\mathrm{ext}}=Z_{e}(s) q=\left(D_{e} s+K_{e}\right) q
$$

In (4), s, $Z_{e}(s), D_{e}$, and $K_{e}$ represent a Laplace operator, environmental impedance, viscous coefficient and spring constant of the environment. Eq. (2) is rewritten by considering modeling error with respect to the variation from the nominal value as follows:

$$
\begin{aligned}
& \left(J_{G n}+\Delta J_{G} s\right) s^{2} q=\tau^{\mathrm{ref}}-\tau^{\text {load }} \\
& =\left(K_{t G n}+\Delta K_{t G}\right) I^{\mathrm{ref}}-\tau^{\text {load }} \\
& J_{G n} s^{2} q=K_{t G n} I^{\mathrm{ref}}-\tau^{\mathrm{ext}}-\tau^{\mathrm{coul}} \\
& \quad-D_{G} \omega-\Delta J_{G} s \omega+\Delta K_{t G} I^{\mathrm{ref}}
\end{aligned}
$$

where $\Delta$ represents a modeling error. From (6), the disturbance torque is defined by including the torque caused by the modeling error in the load torque shown in Eq. (3) as follows:

$$
\tau^{\mathrm{dis}}=\tau^{\mathrm{ext}}+\tau^{\mathrm{coul}}+D_{G} \omega+\Delta J_{G} s \omega-\Delta K_{t G} I^{\mathrm{ref}} .
$$

By using the disturbance torque, (6) is rewritten below.

$$
J_{G n} s^{2} q=\tau^{\mathrm{ref}}-\tau^{\mathrm{dis}}
$$




$$
\tau^{\mathrm{dis}}=\tau^{\mathrm{ref}}-J_{G n} s^{2} q
$$

Equation (9) means that the disturbance torque can be calculated by using reference torque and angular acceleration information. From (9), the disturbance torque is estimated by inserting a Low-pass filter to suppress noise in the high-frequency domain, as follows [6-8]:

$$
\begin{aligned}
\hat{\tau}^{\mathrm{dis}} & =G_{l}^{\mathrm{dis}}(s)\left(\tau_{n}^{\mathrm{ref}}+g_{l}^{\mathrm{dis}} J_{G n} \hat{\omega}\right)-g_{l}^{\mathrm{dis}} J_{G n} \hat{\omega} \\
& =G_{l}^{\mathrm{dis}}(s)\left(\tau_{n}^{\mathrm{ref}}-J_{G n} s \hat{\omega}\right) \\
\tau_{n}^{\mathrm{ref}} & =\tau^{\mathrm{ref}}+\hat{\tau}^{\mathrm{dis}} \\
\hat{\omega} & =\frac{g_{l}^{\mathrm{pd}}}{s+g_{l}^{\mathrm{pd}}} s q \\
G_{l}^{\mathrm{dis}}(s) & =\frac{g_{l}^{\mathrm{dis}}}{s+g_{l}^{\mathrm{dis}}}
\end{aligned}
$$

where $\hat{\bullet}, G_{l}^{\text {dis }}(s), g_{l}^{\text {dis }}, g_{l}^{\mathrm{pd}}$, and subscript $n$ represent an estimated value, low-pass filter (LPF), cut-off frequency of DOB and pseudo differentiation, and a nominal value, respectively. The motion Eq. (8) is rewritten by adding the estimated disturbance $\hat{\tau}^{\text {dis }}$ in reference torque for canceling disturbance as follows:

$$
\begin{aligned}
J_{G n} s^{2} q & =\tau^{\mathrm{ref}}-G_{h}^{\mathrm{dis}}(s) \tau^{\mathrm{dis}}=K_{t G n} I^{\mathrm{ref}}-G_{h}^{\mathrm{dis}}(s) \tau^{\mathrm{dis}} \\
I^{\mathrm{ref}} & =\frac{1}{K_{t G n}} \tau^{\mathrm{ref}}=\frac{1}{K_{t G n}} J_{G n} a^{\mathrm{ref}} \\
G_{h}^{\mathrm{dis}}(s) & =\frac{s}{s+g_{l}^{\mathrm{dis}}}
\end{aligned}
$$

where $a^{\text {ref }}$ and subscript $h$ represent an acceleration reference and a high-pass filter, respectively. From Eq. (15), the following equation is obtained if the bandwidth of the DOB is sufficiently high.

$$
s^{2} q=a^{\mathrm{ref}}-\frac{G_{h}^{\mathrm{dis}}(s)}{J_{G n}} \tau^{\mathrm{dis}} \approx a^{\mathrm{ref}}
$$

As shown in (18), the acceleration response coincides with the acceleration reference, and the robust acceleration controller is realized.

\section{Force control based on force sensor}

Based on (18), the force control system based on force sensor/RTOB is described in the following part.
The acceleration reference of the force sensor-based force control is described as follows:

$$
\begin{aligned}
a^{\mathrm{ref}} & =\frac{1}{J_{f}}\left(\tau^{\mathrm{cmd}}-G_{l}^{\mathrm{ext}}(s) \tau^{\mathrm{sen}}\right) \\
\tau^{\mathrm{sen}} & =\tau^{\mathrm{ext}}+w \\
G_{l}^{\mathrm{ext}}(s) & =\frac{g_{l}^{\mathrm{ext}}}{s+g_{l}^{\mathrm{ext}}}
\end{aligned}
$$

where $J_{f}, w, G_{l}^{\text {ext }}(s)$, superscripts sen and cmd represent a virtual inertia (an inverse of force control gain), sensor noise, LPF for force sensing, sensor value, and command value, respectively. As shown in the above equations, the force sensor based force control system is affected by the force sensor noise. The noise can be suppressed by setting the bandwidth of the LPF lower value. However, the performance and stability of the force control system are deteriorated if the bandwidth of LPF is reduced [7].

\section{Force control based on RTOB}

The external torque can be estimated by applying the structure of the DOB. The external torque is estimated by subtracting the identified friction torque element from (10), as follows:

$$
\begin{aligned}
\hat{\tau}^{\mathrm{ext}} & =G_{l}^{\mathrm{ext}}(s)\left(\tau_{n}^{\mathrm{ref}}+g_{l}^{\mathrm{ext}} J_{G n} \hat{\omega}-\hat{\tau}^{\mathrm{coul}}-\hat{D}_{G n} \hat{\omega}\right)-g_{l}^{\mathrm{ext}} J_{G n} \hat{\omega} \\
& =G_{l}^{\mathrm{ext}}(s)\left(\tau_{n}^{\mathrm{ref}}-J_{G n} s \hat{\omega}-\hat{\tau}^{\mathrm{coul}}-\hat{D}_{G n} \hat{\omega}\right)
\end{aligned}
$$

In this paper, the modeling error of the inertia and the torque constant are assumed to be negligibly small. Additionally, the noise caused by differentiation of position information, including quantization error, is also assumed to be negligibly small. By using the estimated external torque, the motion equation and external torque response of the RTOB-based force control with DOB are described as follows:

$$
\begin{aligned}
& s^{2} q=\frac{1}{J_{f}}\left(\tau^{\mathrm{cmd}}-\hat{\tau}^{\mathrm{ext}}\right) \\
& =\frac{1}{J_{f}}\left(\tau^{\mathrm{cmd}}-G_{l}^{\mathrm{ext}}(s) \tau^{\mathrm{ext}}\right)+\frac{1}{J_{f}} G_{l}^{\mathrm{ext}}(s) \\
& \left(\Delta \tau^{\mathrm{coul}}+\Delta D_{G n} \omega\right) \\
& G_{l}^{\mathrm{ext}}(s) \tau^{\mathrm{ext}}=\tau^{\mathrm{cmd}}-J_{f} s^{2} q+G_{l}^{\mathrm{ext}}(s)\left(\Delta \tau^{\mathrm{coul}}+\Delta D_{G n} \omega\right) .
\end{aligned}
$$


As shown in (26), the force control system based on RTOB is affected by modeling error of the coulomb friction and viscous friction. Especially, the precise modeling of the coulomb friction is difficult.

\section{Proposed external torque system based on integration of the force sensor and RTOB}

As shown in the last section, the force control system is affected by the force sensor noise or modeling error of the friction torque. This section describes the proposed force control system based on the integration of the force sensor and RTOB. The proposed method can simultaneously achieve suppressing the force sensor noise and reducing the effect of modeling error of friction torque.

\section{Structure of the proposed external torque estimation}

The Coulomb friction can be assumed to exist in the lower frequency domain, and the sensor noise can be considered to exist in the higher frequency domain. Therefore, the proposed method effectively uses the high-frequency component of RTOB and the low-frequency component of the force sensor.

The higher frequency domain of the external torque is extracted by using RTOB-based structure as follows:

$$
\begin{aligned}
G_{h}^{I}(s) \tau^{\mathrm{obs}} & =G_{h}^{I}(s)\left(\tau_{n}^{\mathrm{ref}}-J_{G n} s \hat{\omega}-D_{G n} \hat{\omega}\right) \\
& =G_{h}^{I}(s) \tau^{\mathrm{ext}}-G_{h}^{I}(s)\left(\Delta D_{G} \omega-\tau^{\mathrm{coul}}\right) \\
G_{h}^{I}(s) & =\frac{s}{s+g^{I}}
\end{aligned}
$$

where $G_{h}^{I}(s)$ and $g^{I}$ represent the high-pass filter and its bandwidth for the integration of the force sensor and RTOB information. As shown in (27), the proposed method uses only the model of the viscous coefficient as the model of friction.

On the other hand, the low-frequency component of the external torque is obtained by using LPF $G_{l}^{I}(s)$ described below.

$$
\begin{aligned}
G_{l}^{I}(s) \tau^{\mathrm{sen}} & =G_{l}^{I}(s) \tau^{\mathrm{ext}}+G_{l}^{I}(s) w \\
G_{l}^{I}(s) & =\frac{g^{I}}{s+g^{I}}
\end{aligned}
$$

A summation of Eqs. (28) and (30) are shown below:

$$
\begin{aligned}
G_{l}^{I}(s) \tau^{\mathrm{sen}}+G_{h}^{I}(s) \tau^{\mathrm{obs}}= & G_{l}^{I}(s) \tau^{\mathrm{ext}}+G_{h}^{I}(s) \tau^{\mathrm{ext}} \\
& -G_{h}^{I}(s)\left(\Delta D_{G} \omega-\tau^{\mathrm{coul}}\right)+G_{l}^{I}(s) w
\end{aligned}
$$

$$
=\tau^{\mathrm{ext}}-G_{h}^{I}(s)\left(\Delta D_{G} \omega-\tau^{\mathrm{coul}}\right)+G_{l}^{I}(s) w .
$$

From Eq. 33, the external torque can be estimated through $\mathrm{LPF} G_{l}^{\text {ext }}(s)$ as follows:

$$
\begin{aligned}
\hat{\tau}^{\mathrm{ext}}= & G_{l}^{\mathrm{ext}}(s)\left(G_{l}^{I}(s) \tau^{\mathrm{sen}}+G_{h}^{I}(s) \tau^{\mathrm{obs}}\right) \\
= & G_{l}^{\mathrm{ext}}(s) \tau^{\mathrm{ext}}-G_{l}^{\mathrm{ext}}(s) G_{h}^{I}(s)\left(\Delta D_{G} \omega-\tau^{\mathrm{coul}}\right) \\
& +G_{l}^{\mathrm{ext}}(s) G_{l}^{I}(s) w .
\end{aligned}
$$

Figure 2 shows the block diagram of the proposed method based on (34). As shown in (35), the friction torque in RTOB information and force sensor noise are suppressed by $G_{l}^{\text {ext }}(s) G_{h}^{I}(s)$ and $G_{l}^{\text {ext }}(s) G_{l}^{I}(s)$, respectively. As a result, the external torque is estimated through the LPF $G_{l}^{\text {ext }}(s)$. Figure 3 shows the bode diagrams of the external torque estimation with respect to proposed method $G_{l}^{\text {ext }}(s)$, information from RTOB $G_{l}^{\text {ext }}(s) G_{h}^{I}(s)$, and force sensor information $G_{l}^{\text {ext }}(s) G_{l}^{I}(s)$.

\section{Application to force control system}

Figure 4 shows the force control system based on the proposed method. From Fig. 4 the motion equation of the proposed method is described below.

$$
\begin{aligned}
s^{2} q= & \frac{1}{J_{f}}\left(\tau^{\mathrm{cmd}}-G_{l}^{\mathrm{ext}}(s) \tau^{\mathrm{ext}}\right) \\
& +\frac{1}{J_{f}} G_{l}^{\mathrm{ext}}(s) G_{h}^{I}(s)\left(\tau^{\mathrm{coul}}+\Delta D_{G n} \omega\right)-\frac{1}{J_{f}} G_{l}^{\mathrm{ext}}(s) G_{l}^{I}(s) w
\end{aligned}
$$

From (36), the response of the external torque is derived as follows:

$$
\begin{aligned}
\hat{\tau}^{\mathrm{ext}}= & \tau^{\mathrm{cmd}}-J_{f} s^{2} q+G_{l}^{\mathrm{ext}}(s) G_{h}^{I}(s)\left(\tau^{\mathrm{coul}}+\Delta D_{G n} \omega\right) \\
& -G_{l}^{\mathrm{ext}}(s) G_{l}^{I}(s) w .
\end{aligned}
$$

Because the friction torque and sensor noise are suppressed by the $G_{l}^{\text {ext }}(s) G_{h}^{I}(s)$ and $G_{h}^{\text {ext }}(s) G_{l}^{I}(s)$ and the inertia torque $J_{f} s^{2} q$ converges to zero in a steady-state, the external torque response converges to the command torque as follows:

$$
\hat{\tau}^{\mathrm{ext}} \approx \tau^{\mathrm{cmd}} .
$$

As a result, the performance of the force control system is improved by the proposed method.

\section{Application to the bilateral control system}

The proposed external torque estimation is applied to the bilateral control system as one of the position/ force hybrid control [12-14]. By applying the proposed 


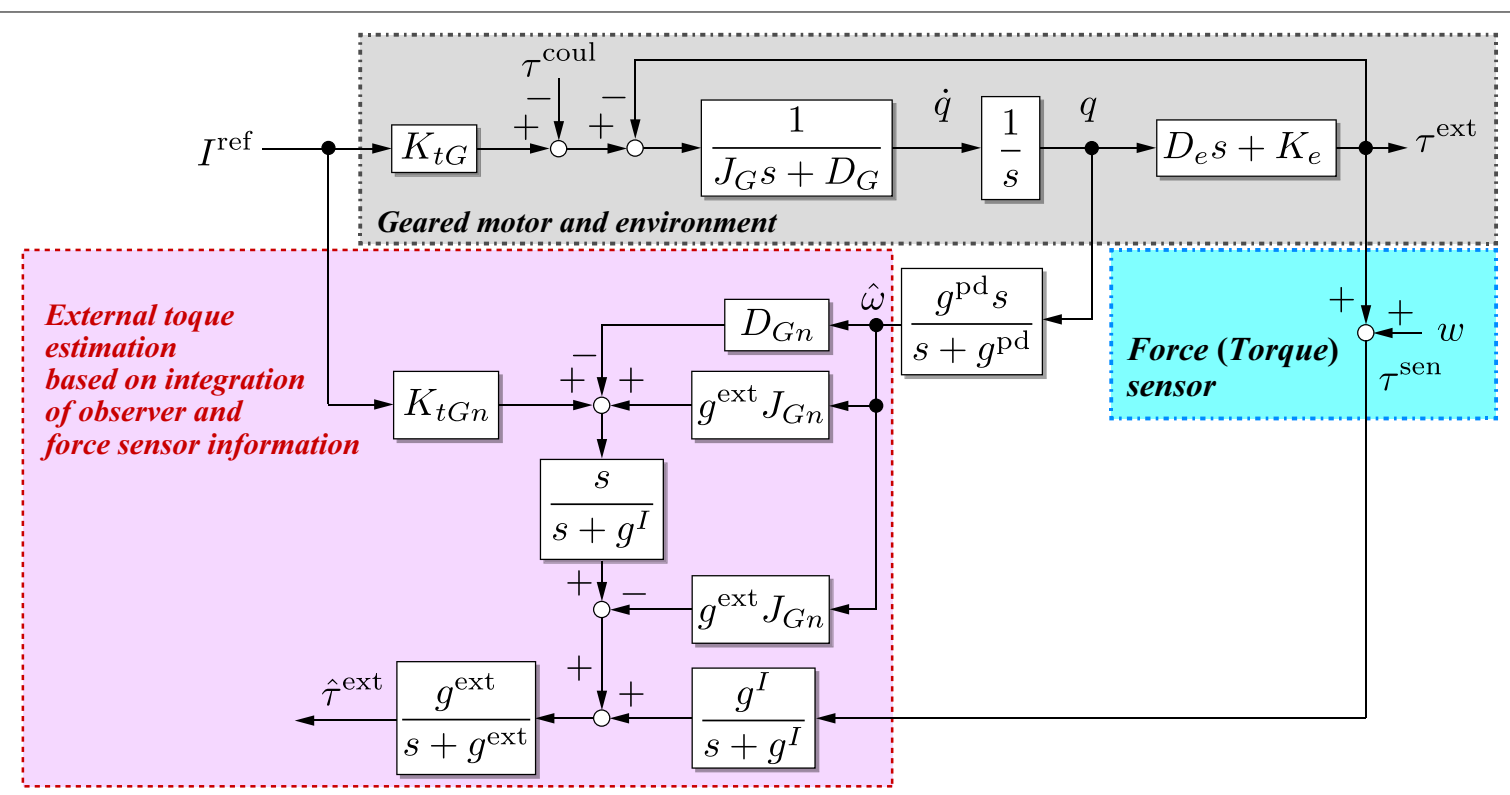

Fig. 2 Block diagram of the proposed external torque estimation

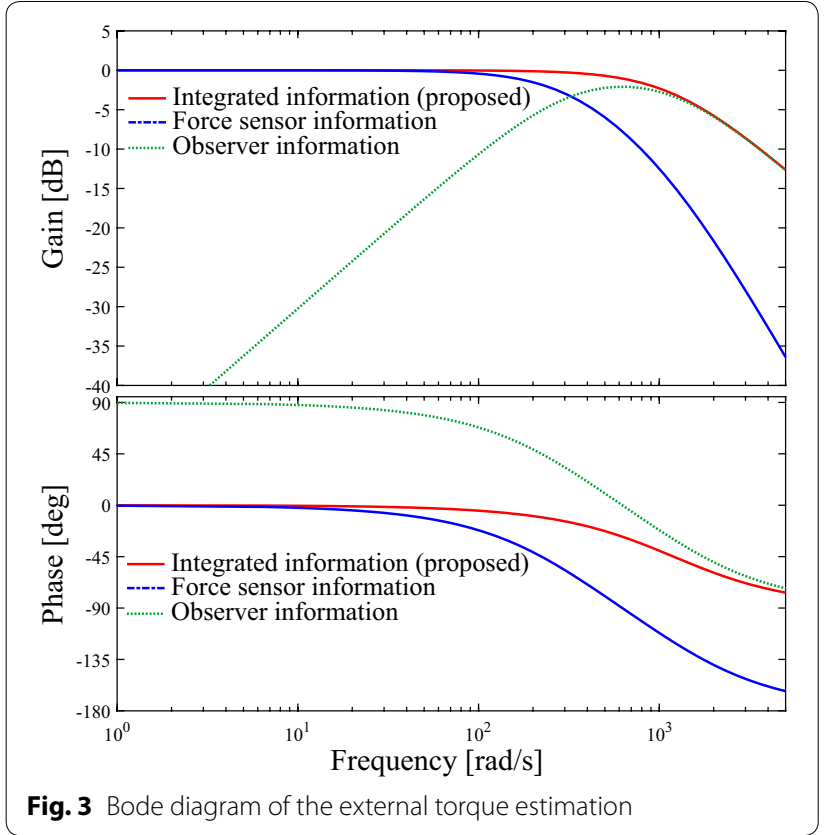

method, the operability of the bilateral control system can be improved because friction torque which behaves as an operational torque can be compensated. The control goals of bilateral control are described as follows:

$$
\begin{aligned}
& \hat{\tau}_{F}^{\text {ext }}=\hat{\tau}_{m}^{\text {ext }}+\hat{\tau}_{s}^{\text {ext }}=\tau_{F}^{\mathrm{cmd}}=0 \\
& q_{X}=q_{m}-q_{s}=q_{X}^{\mathrm{cmd}}=0
\end{aligned}
$$

where subscripts $m, s, X$, and $F$ represent a master system, a slave system, variables of the position control, and the force control, respectively. The control goal of force control represents the realization of the law of action and reaction. On the other hand, the control goal of the position control represents the synchronization of master and slave position. The acceleration reference vector $\boldsymbol{a}^{\text {ref }}$ for the bilateral control system is obtained by using the inverse transformation matrix of $\boldsymbol{T}$ as follows [12-14]:

$$
\begin{gathered}
\boldsymbol{a}^{\mathrm{ref}}=\left[\begin{array}{c}
a_{m}^{\mathrm{ref}} \\
a_{s}^{\mathrm{ref}}
\end{array}\right]=\boldsymbol{T}^{-1}\left[\begin{array}{c}
a_{F}^{\mathrm{ref}} \\
a_{X}^{\mathrm{ref}}
\end{array}\right]=\frac{1}{2}\left[\begin{array}{cc}
1 & 1 \\
1 & -1
\end{array}\right]\left[\begin{array}{c}
a_{F}^{\mathrm{ref}} \\
a_{X}^{\mathrm{ref}}
\end{array}\right] \\
{\left[\begin{array}{c}
a_{F}^{\mathrm{ref}} \\
a_{X}^{\mathrm{ref}}
\end{array}\right]=\left[\begin{array}{c}
C_{p}(s)\left(q_{X}^{\mathrm{cmd}}-q_{X}\right) \\
\frac{1}{J_{f}}\left(\hat{\tau}_{F}^{\mathrm{cmd}}-\hat{\tau}_{F}^{\mathrm{ext}}\right)
\end{array}\right]=\left[\begin{array}{c}
-\left(K_{v} s+K_{p}\right) q_{X} \\
-\frac{1}{J_{f}} \hat{\tau}_{F}^{\text {ext }}
\end{array}\right]}
\end{gathered}
$$

where $C_{p}(s)=K_{v} s+K_{p}$ represents a PD controller of position control system. Figure 5 shows the block diagram of the bilateral control system with the proposed method.

\section{Analysis}

This section describes the performance and stability analysis of the proposed method. The frequency characteristics of the response characteristics, the disturbance suppression performance, and the noise sensitivity are analyzed. Additionally, the stability with the variation of design parameters is analyzed from the viewpoint of root locus. Table 1 lists the parameters used in the analysis. 


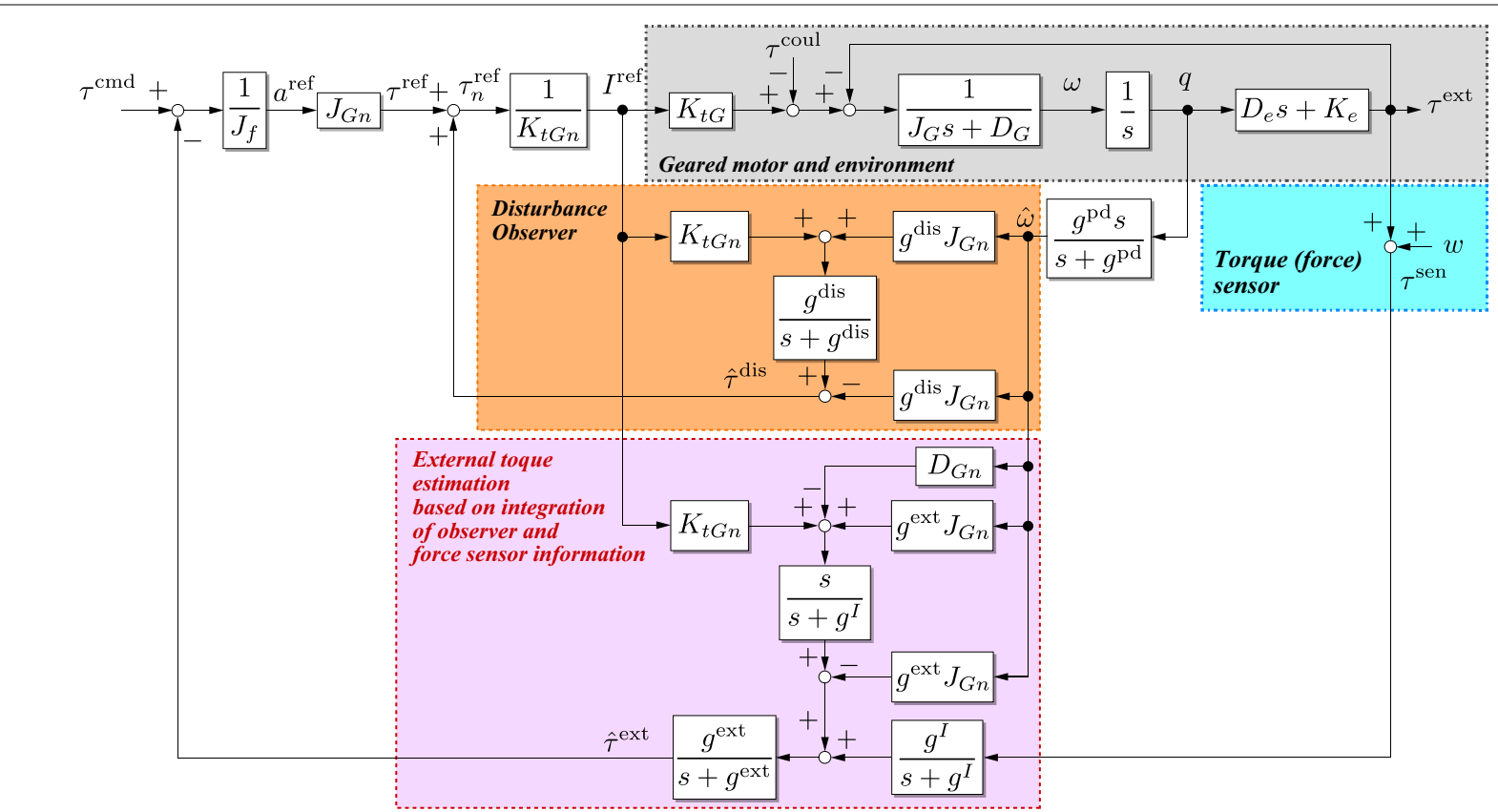

Fig. 4 Block diagram of the proposed method

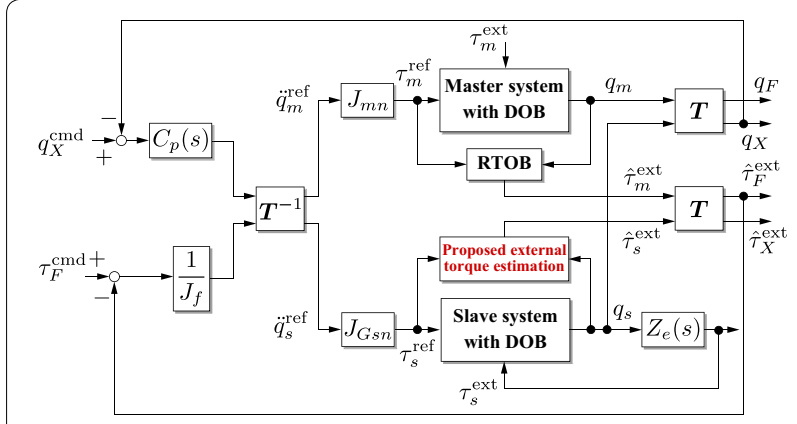

Fig. 5 Block diagram of a bilateral control system with the proposed external force estimation

Table 1 Parameters used in the analysis

\begin{tabular}{lll}
\hline Parameter & Description & Value \\
\hline$G_{r}$ & Gear ratio & 30 \\
$J_{n}$ & Nominal motor inertia & $1.59 \times 10^{-6}$ \\
$K_{t n}$ & Nominal torque constant & $6.5 \times 10^{-2} \mathrm{Nm} / \mathrm{A}$ \\
$D$ & Nominal viscosity & $2.2 \times 10^{-3} \mathrm{Nms} / \mathrm{rad}$ \\
$K_{e}$ & Environmental stiffness & $1.0 \times 10^{4} \mathrm{Nm} / \mathrm{rad}$ \\
$D_{e}$ & Environmental viscosity & $0 \mathrm{Nms} / \mathrm{rad}$ \\
$J_{f}$ & Virtual mass gain & $1.0 \times 10^{-3} \mathrm{kgm}^{2}$ \\
$g^{\text {dis }}$ & Bandwidth of DOB & $600 \mathrm{rad} / \mathrm{s}$ \\
$g^{\text {ext }}$ & Bandwidth of RTOB & $1200 \mathrm{rad} / \mathrm{s}$ \\
$g^{\text {pd }}$ & Bandwidth of pseudo-differential & $1200 \mathrm{rad} / \mathrm{s}$ \\
$g_{l}$ & Bandwidth of integration & $325 \mathrm{rad} / \mathrm{s}$ \\
\hline
\end{tabular}

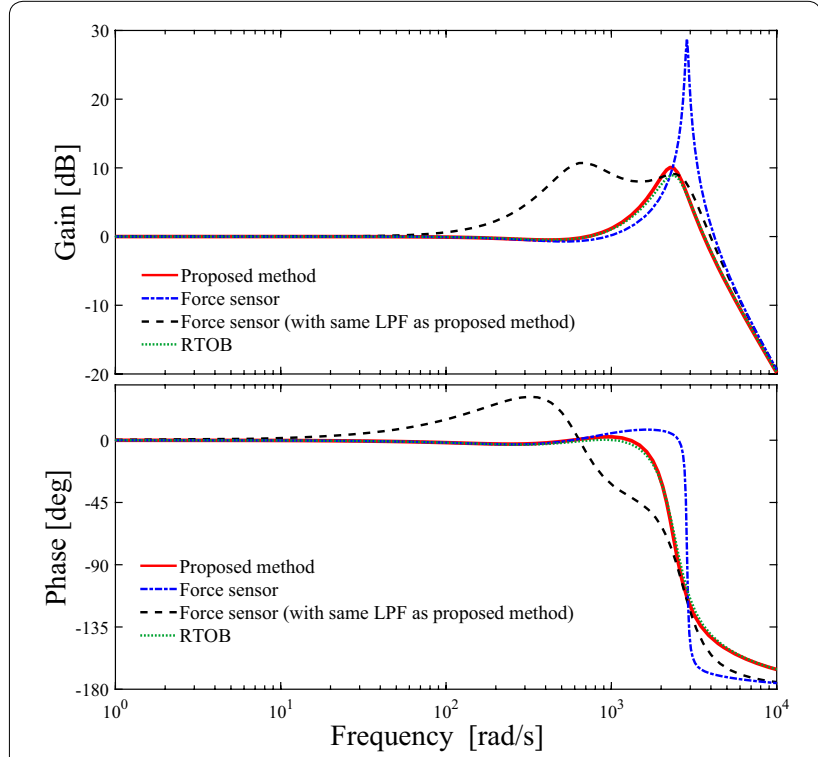

Fig. 6 Bode diagram of the transfer functions from command torque to output external torque

\section{Performance analysis}

Figure 6 shows the bode diagrams of the transfer functions from command torque to output (external) torque which is derived from Fig. 4. This figure illustrates the characteristics of the proposed method, the RTOB-based method, the force sensor-based method, and the force 
sensor-based method with the same LPF for the torque sensor as the proposed method $\left(G_{l}^{\text {ext }}(s) G_{l}^{I}(s)\right)$. As shown by the figure, the bandwidths of these methods are almost same. However, the peak value in the gain diagram of the proposed method is smaller than that of the force sensorbased methods. Additionally, it can be found that the proposed method and the RTOB-based method show similar response characteristics in both gain and phase characteristics.

Figure 7 shows the bode diagram of the transfer functions from load torque to output torque. It is found that the RTOB-based method cannot attenuate the lowerfrequency component of the load torque. Therefore, the friction torque should be identified precisely. Conversely, Fig. 7 shows that the proposed method can suppress the lower-frequency component of the load torque including the friction torque without its identification. However, the force sensor-based method does not use the highfrequency components of the RTOB, so that the disturbance suppression characteristics are higher than that of the proposed method. However, the force sensor-based method also has a peak value in the disturbance response characteristics. Also, it is found that disturbance beyond the band of external force estimation $(1200 \mathrm{rad} / \mathrm{s}$ in this case) is suppressed.

Figure 8 shows the bode diagram of noise sensitivity with respect to the force sensor-based methods and the proposed method. As shown in the figure, the noise suppression performance of the proposed method is better than that of the force sensor-based method. On the other

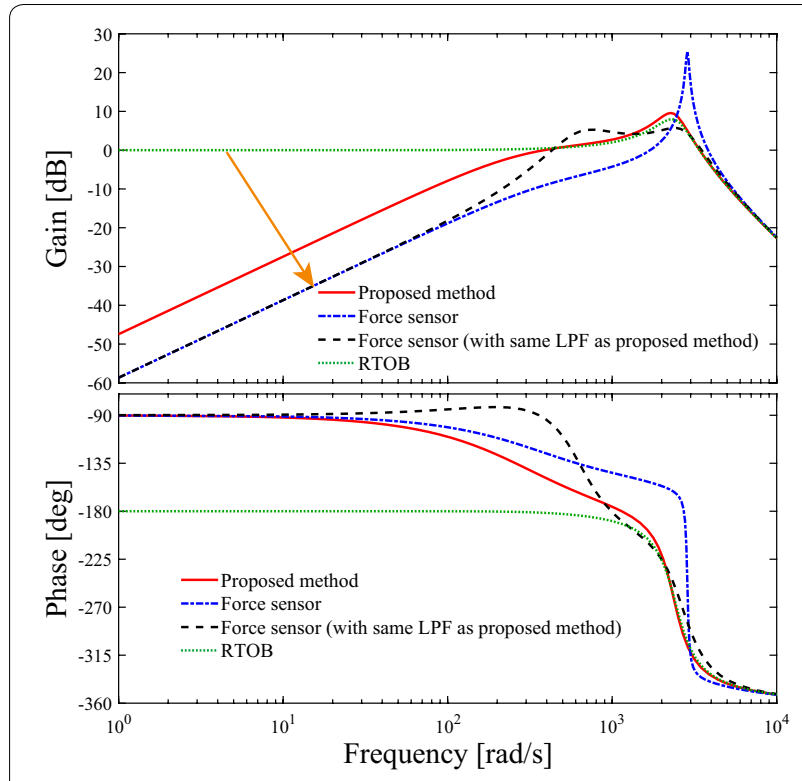

Fig. 7 Bode diagram of the transfer functions from load torque to output external torque

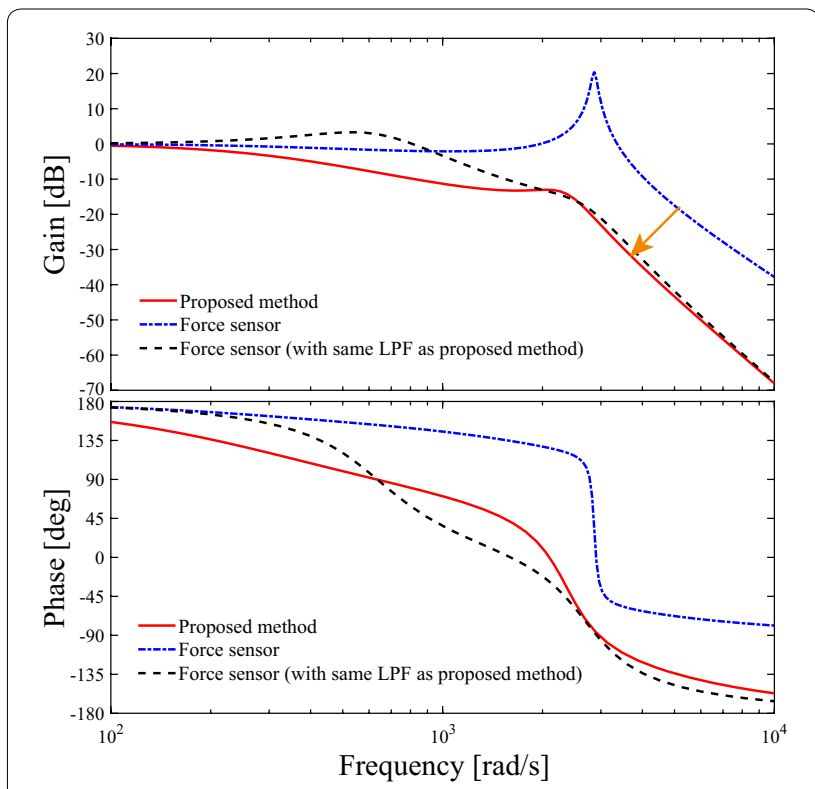

Fig. 8 Bode diagram of the transfer functions from sensor noise to output external torque

hand, the force sensor-based method with the same LPF as the proposed method $\left(G_{l}^{\text {ext }}(s) G_{l}^{I}(s)\right)$ also improves the noise suppression performance. However, the response characteristics of this method deteriorate compared to the proposed method as shown in Fig. 6.

Figure 9a shows the bode diagram of the response characteristics in the proposed method with the variation of $g^{I}$ from 100 to $1100 \mathrm{rad} / \mathrm{s}$. This figure shows that the bandwidth variation for sensor and RTOB integration has almost no effect on the bandwidth of response characteristics, though the peak value of the gain diagram is increased. Figure $9 \mathrm{~b}$ shows the bode diagram of the disturbance suppression characteristics with the variation of $g^{I}$. From Fig. 9b, it can be confirmed that the disturbance suppression characteristics improve as $g^{I}$ increases. In contrast, Fig. 9c shows that noise sensitivity increases as $g^{I}$ increases. From these results, there is a trade-off between the load torque (friction torque) suppression and noise sensitivity in the proposed method.

The above performance comparison is summarized as follows:

- The proposed method not only has the same response characteristics as the RTOB-based method but also can compensate for the Coulomb friction torque without identification.

- The noise suppression characteristics of the proposed method are superior to the force sensorbased method. 


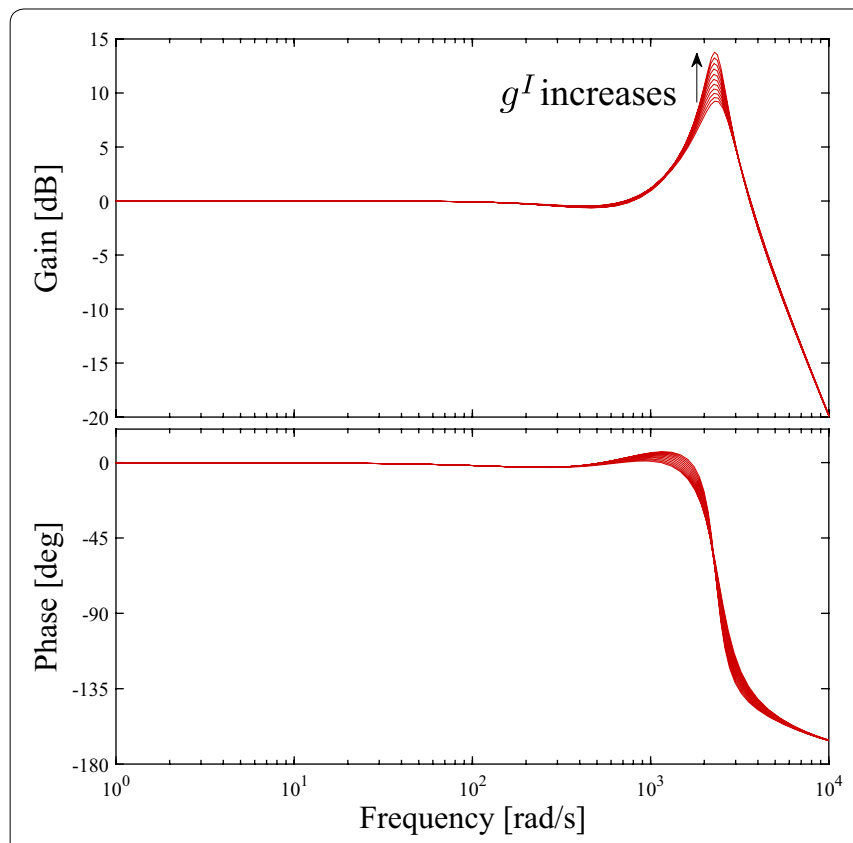

a

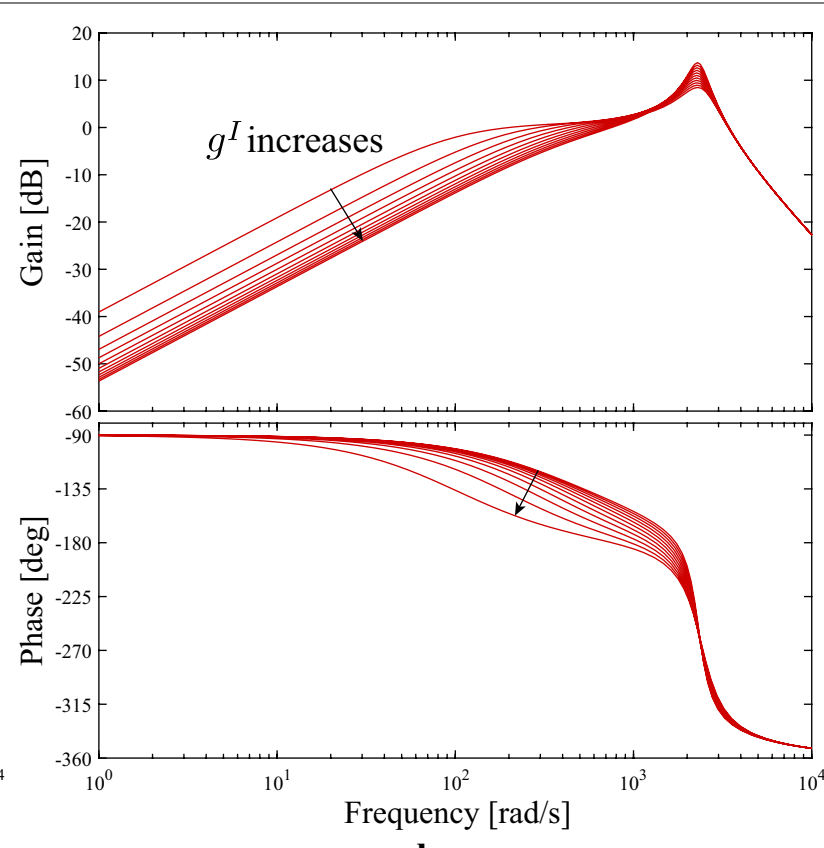

b

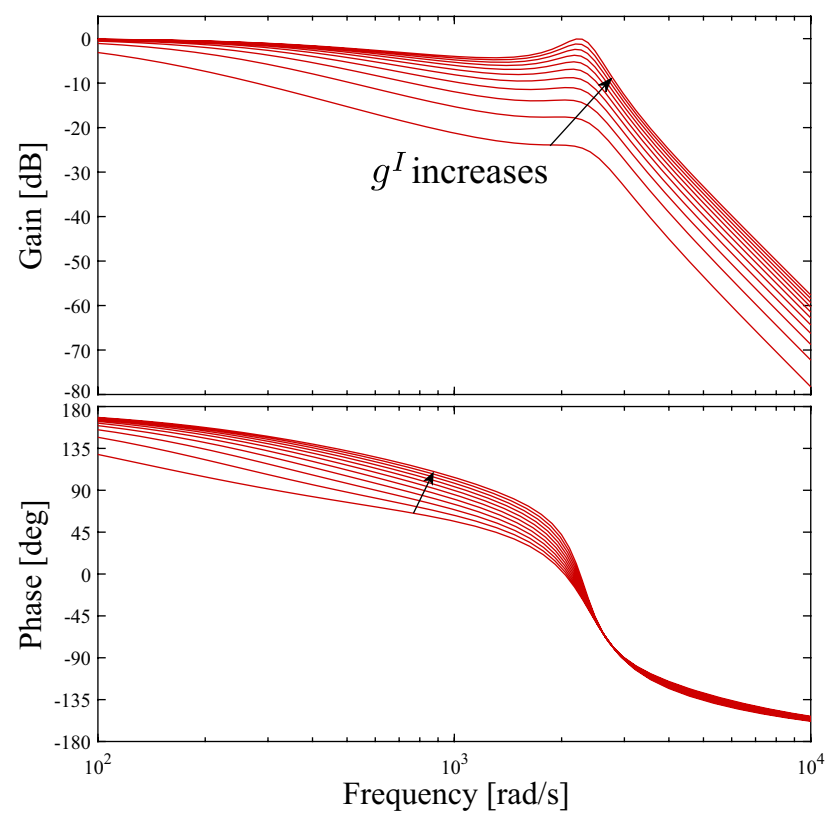

c

Fig. 9 Bode diagram of transfer functions with the variation of the bandwidth of sensor and RTOB integration $g^{\prime}$ from 100 to $1100 \mathrm{rad} / \mathrm{s}$. a Transfer functions from command torque to output external torque. $\mathbf{b}$ Transfer functions from load torque to output external torque. $\mathbf{c}$ Transfer functions from sensor noise to output external torque

- There is a trade-off between the noise suppression performance due to the force sensor and the friction-torque suppression performance, depending on the bandwidth $g^{I}$.
From the above, the proposed method can suppress the effects of friction torques while suppressing noise due to the force sensor if the noise due to the reaction force estimation using the position information is sufficiently 


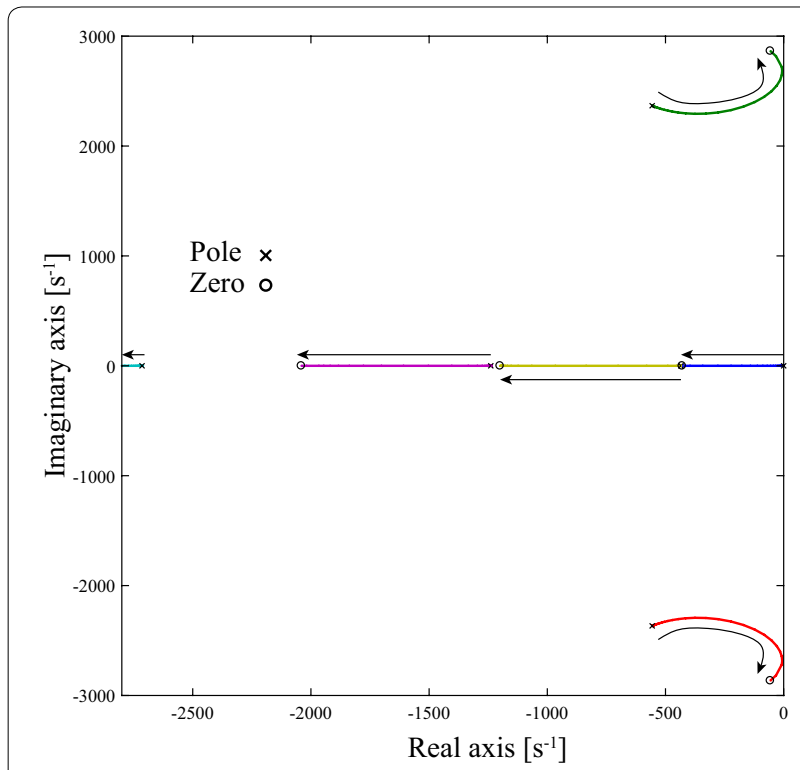

Fig. 10 Root locus for variation of sensor and RTOB information integration bandwidth $g^{\prime}$

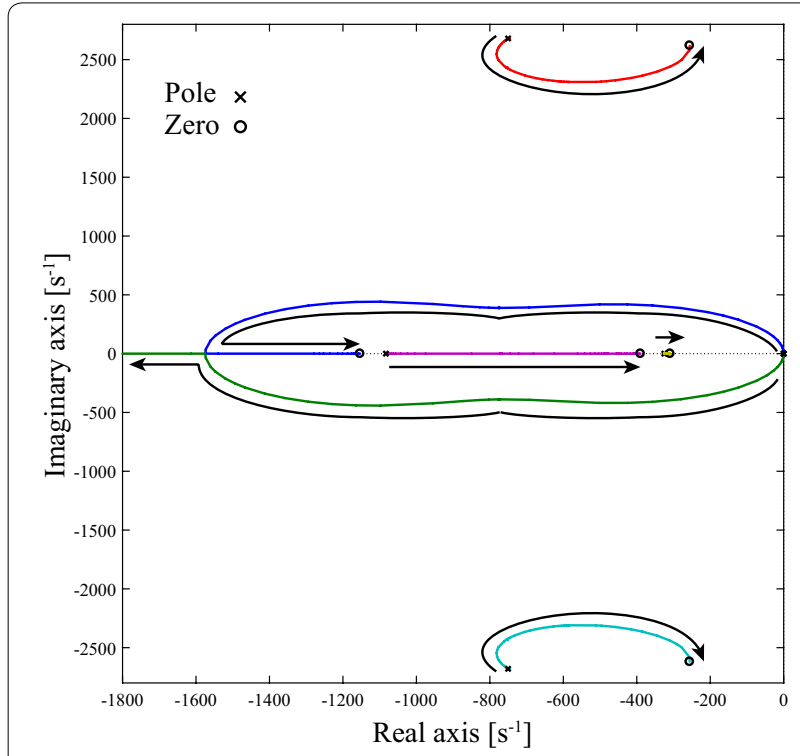

Fig. 11 Root locus for variation of sensor and RTOB bandwidth $g^{\text {ext }}$

small (in other words, if the resolution of the position sensor is sufficiently high).

\section{Stability analysis}

Figures 10 and 11 show the root locus for variation of sensor and RTOB information integration bandwidth $g^{I}$ and the root locus for variation of sensor and RTOB

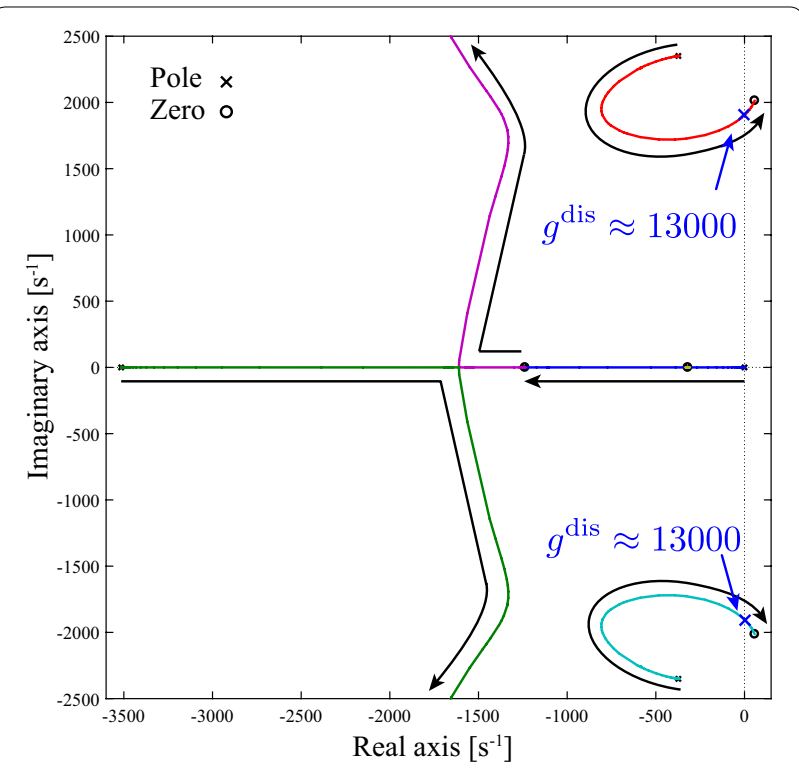

Fig. 12 Root locus for variation of DOB bandwidth $g^{\text {dis }}$

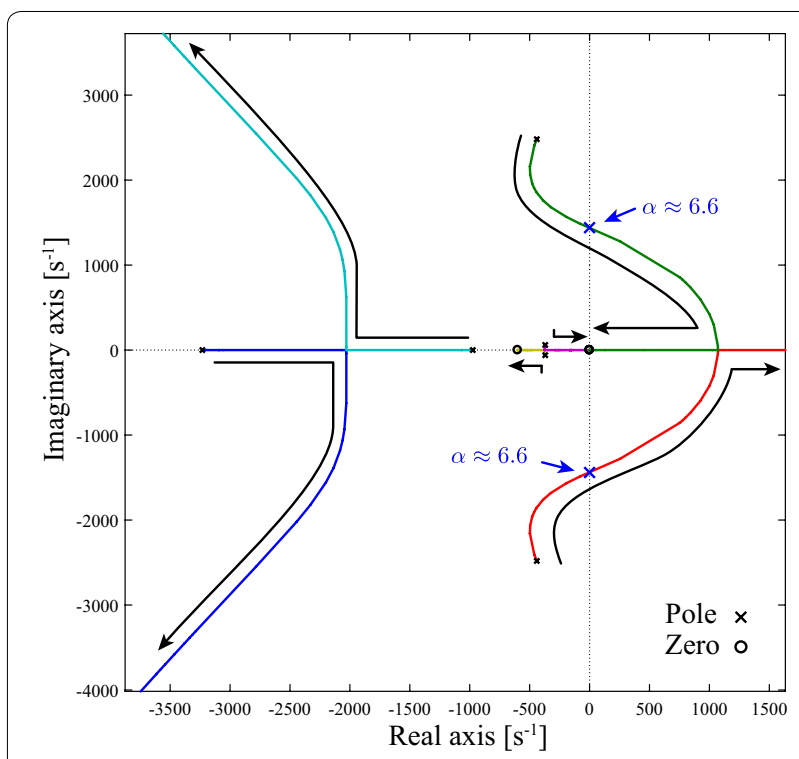

Fig. 13 Root locus for variation of the modeling error about viscous friction

bandwidth $g^{\text {ext }}$. These figures represent that $g^{I}$ and $g^{\text {ext }}$ do not affect the stability.

Figure 12 shows the root locus for variation of the bandwidth of DOB $g$ dis. As shown in the figure, the proposed system becomes unstable if the $g^{\text {dis }}$ is set larger than 13,000 $\mathrm{rad} / \mathrm{s}$. In practical cases, however, the acceleration control system works well even if $g$ dis is set to a lower value than that value. 
Figure 13 shows the root locus for variation of the modeling error about viscous coefficient of the actuator. The modeling error is expressed by error ratio $\alpha$ as $D_{n}=\alpha D(\alpha>0)$. As shown in the figure, the proposed system becomes unstable if the $\alpha$ is larger than about 6.6. From this result, it is found that the nominal value should be set a smaller if the model of the viscosity is uncertain.

\section{Experiments}

This section describes the experiments for the evaluation of the proposed method. The proposed method, force sensor-based method, and RTOB based method are compared in the force control system and bilateral control system.

\section{Experimental setup}

Figure 14 shows the experimental setup. The single DOF geared motor shown in Fig. 14a is used for experiments of the force control, and for the slave system in bilateral control. The geared motor is composed by a brush-less DC motor (direct drive (DD) motor: MDS-3018, MICROTECH LABORATORY INC.) with a harmonic gear (CSF17-30-2UH, HARMONIC DRIVE SYSTEMS INC.). The
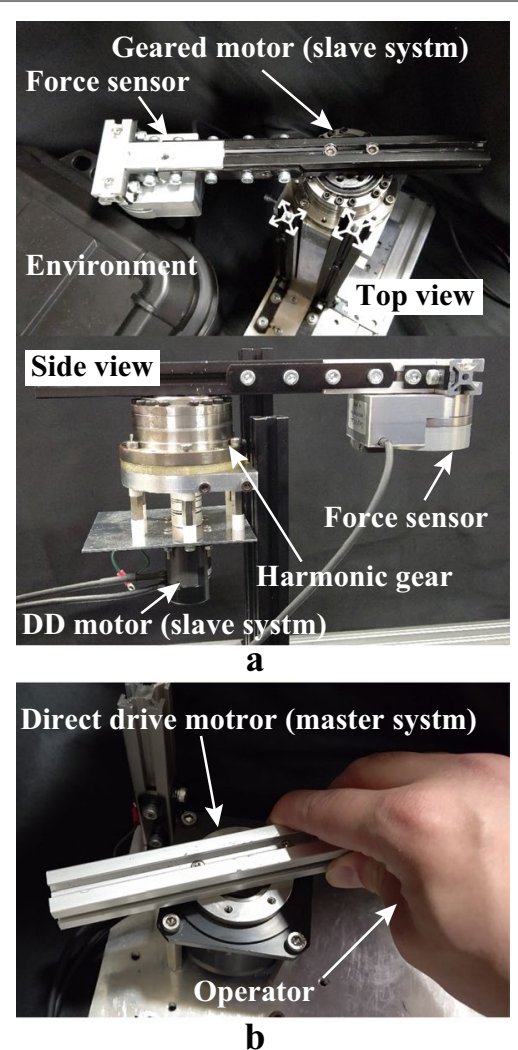

Fig. 14 Experimental setup. a Geared motor (slave system). b Direct drive motor (master system) harmonic drive gear causes the friction torque, though the backlash is small. The DD motor (MDH-7018, MICROTECH LABORATORY INC.) shown in Fig. 14b is used for the master system in the bilateral control experiment. In this experiment, the friction inside of the DD motors is assumed to be negligibly small. Therefore, external torque added to the master system is estimated only by using an RTOB. The parameter variation of the motor inertia and torque constant from nominal value also assumed to be negligible in this experiment.

The actuators are controlled by a desktop computer with Linux both in the force control and bilateral control experiments. The angle information of the actuators is measured by the position encoder and obtained through a pulse counter. The q-axis current references are calculated in the computer and the reference value is inputted to servo drivers for the DD motors through a DA converter.

A six-axis force sensor (PFS055YA251G6, Leptrino Inc.) with an amplifier module is mounted at the tip of the arm in the geared motor shown in Fig. 14a. The rated force of the sensor is $\pm 250 \mathrm{~N}$ and its resolution is $1 / 2000$. The output voltage of the force sensor is taken into the computer via an $\mathrm{AD}$ converter and converted into a force dimension. The value of the external torque is obtained by multiplying the value of one axis of the force sensor by the length of the slave arm $l_{s}$ as $\tau^{\text {sen }}=l_{s} G_{l}^{\text {ext }}(s) F^{\text {ext }}$. In this paper, the only one-degree-of-freedom operation is dealt with for simplicity. However, the proposed method can be applied without problems to a multi-degree-of-freedom system by considering the Jacobian matrix and homogeneous transformation matrix of the manipulator. As for the case of multi-degree-of-freedom manipulator control in the workspace, the proposed method is applied by integrating the force information converted from the external torque estimated by RTOB in the joint space into the workspace and the information of the force sensor.

A box which is shown in Fig. 14a is used as an environment. The geared motor performs contact motions with the environment both in the experiments of force control and bilateral control. In the bilateral control experiment, a human operator moves the master arm and performs free motions and contact motions.

This part also explains the procedures for obtaining the friction characteristics. In this paper, the viscous coefficient and Coulomb friction are assumed to be constant values. From (2) and (3), if the step current $I^{\text {ss }}$ signal is inputted to the actuator, the response of the motor side of the actuator in a steady state is described below.

$$
\lim _{s \rightarrow 0} s \cdot K_{t} \frac{I^{\mathrm{ss}}}{s}=\lim _{s \rightarrow 0}(J s+D) \omega_{M}+\tau_{M}^{\text {coul }}=D \omega_{M}^{\mathrm{ss}}+\tau_{M}^{\text {coul }}
$$




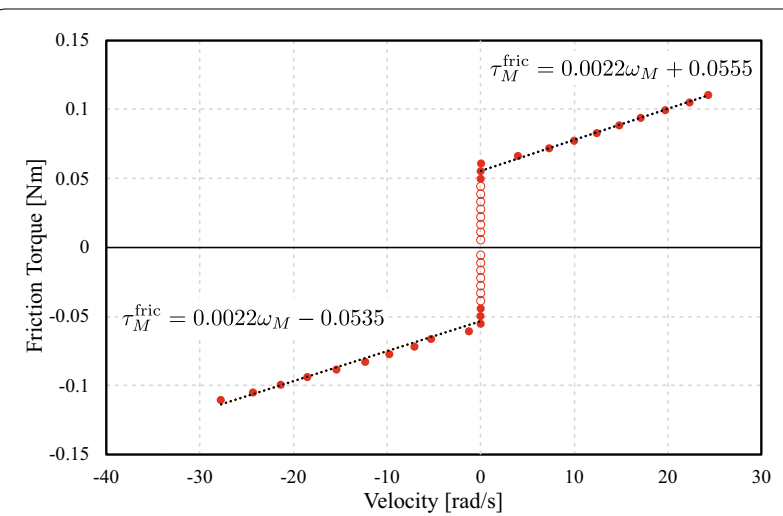

Fig. 15 Result of friction identification

Table 2 Parameters used in the experiments

\begin{tabular}{lll}
\hline Parameter & Description & Value \\
\hline$T_{s}$ & Sampling time & $0.75 \mathrm{~ms}$ \\
$\delta q_{s}$ & Resolution of encoder at slave-side & $108,000 \mathrm{pulse} / \mathrm{rev}$. \\
$\delta q_{m}$ & Resolution of encoder at master-side & $324,000 \mathrm{pulse} / \mathrm{rev}$. \\
$J_{m n}$ & Nominal motor inertia of master system & $0.94 \times 10^{-6}$ \\
$K_{t m n}$ & Nominal torque constant of master & $0.3 \mathrm{Nm} / \mathrm{A}$ \\
& $\quad$ system & $0.155 \mathrm{~m}$ \\
$I_{s}$ & Arm length of slave system & 2000 \\
$K_{p}$ & Position gain & 127 \\
$K_{v}$ & Velocity gain &
\end{tabular}

where superscript ss and subscript $M$ represent a steady-state variable and a motor-side variable, respectively. From (43), the relationship between the motor torque, motor angular velocity, and Coulomb friction is expressed as follows:

$$
K_{t} I^{\mathrm{ss}}=\tau_{M}^{\mathrm{ss}}=D \omega_{M}^{\mathrm{ss}}+\tau_{M}^{\mathrm{coul}} .
$$

From (44), the viscous coefficient and coulomb friction modeled as constant values are obtained by the leastsquares method using the step motor torque (current) and the angular velocity information. Figure 15 shows the identification result of the friction torque. The friction is identified by using the data represented by the filled circles in Fig. 15. As for the angular-velocity information, average values for $2.5 \mathrm{~s}$ are used as a steady-state value. The identified friction torque is described as follows:

$$
\tau_{M}^{\text {fric }}= \begin{cases}0.0022 \omega_{M}+0.0555 & \left(\omega_{M} \geq 0\right) \\ 0.0022 \omega_{M}-0.0535 & \left(\omega_{M}<0\right) .\end{cases}
$$

Table 2 lists the parameters used in the experiments. The other parameters are the same as those listed in Table 1.

\section{Experimental results of force control}

Figure 16a shows the experimental result of the forcesensor based force control. As shown in the figure, a hunting phenomenon is observed in the transient state. This figure represents that the force sensor-based force controller is difficult to achieve stable contact motions in this condition.

Figure 16b shows the experimental result of the RTOBbased force control. As shown in the figure, the reason why the output of RTOB is generated even though the value of the force sensor is almost zero is that the value of the friction torque is outputted. These results show that the RTOB-based method cannot move because of the influence of friction.

Conversely, Fig. 16c represents that the proposed method achieves noise suppression and friction compensation simultaneously. As a result, the proposed method can achieve stable contact motions. The shaded area in Fig. 16c shows the step-wisely response. The indicated torque corresponds to the friction torque that could not be compensated because the bandwidth of the sensor and observer integration $g^{I}$ is finite. If the band for the integration can be increased, the remained friction torque can be compensated. Also, if the command torque has a larger value, the effect of remained friction torque becomes relatively small. However, increasing this bandwidth means that the influence of the noise from the force sensor also increases. This is because of the tradeoff relationship shown in Fig. 9b, c.

\section{Experimental results of bilateral control}

Figure $17 \mathrm{a}-\mathrm{c}$ show the experimental results of bilateral control system based on RTOB, force sensor, and the proposed method, respectively. In order to make the results easier to see, the external torque on the master side is inverted and displayed in these figures. Figure 17a shows that the human operator cannot perform both of the free motions and contact motions due to the large operational torque caused by the identification error of the friction torque.

On the other hand, the force sensor-based method and the proposed method achieves both of the free motions and contact motions with realizing the law of action and reaction as shown in Fig. 17b, c. As suggested in Fig. 7, the operational torque generated during free motion due to the frictional torque is larger in the proposed method than in the force sensor-based method. However, it is clear that the proposed method attenuates the friction torque much more than the RTOB-based method.

As shown in Fig. 18a, the torque response of force sensor-based method is oscillating due to the sensor noise and the resulting position response is also vibrating. 

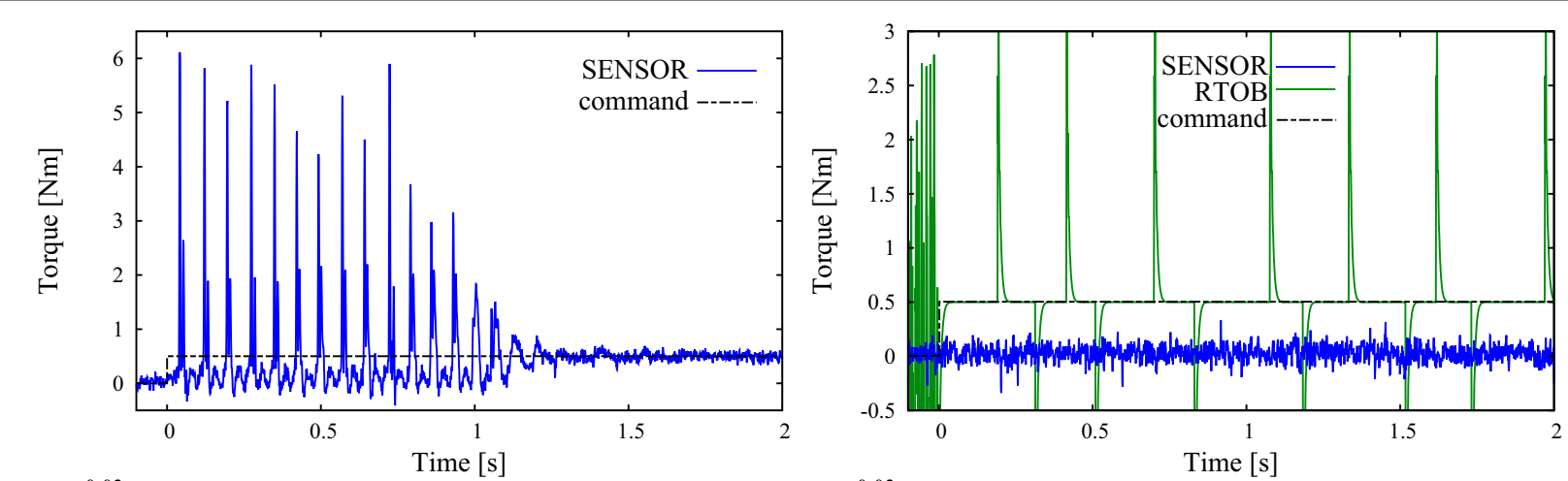

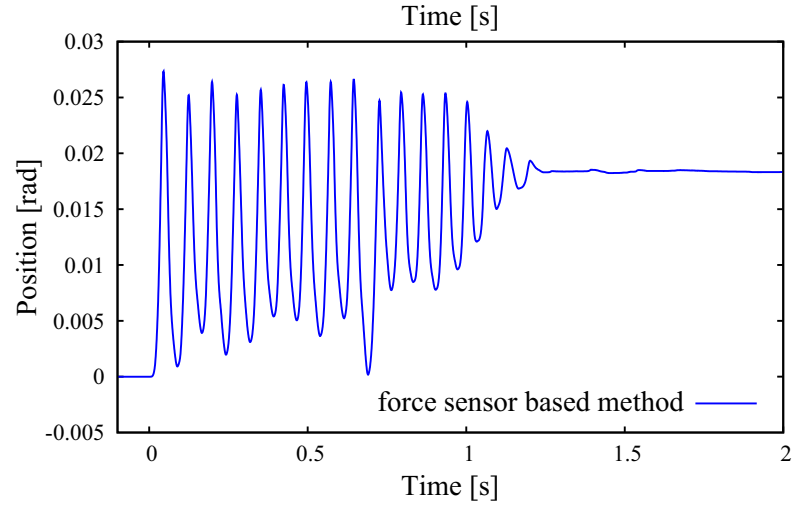

a

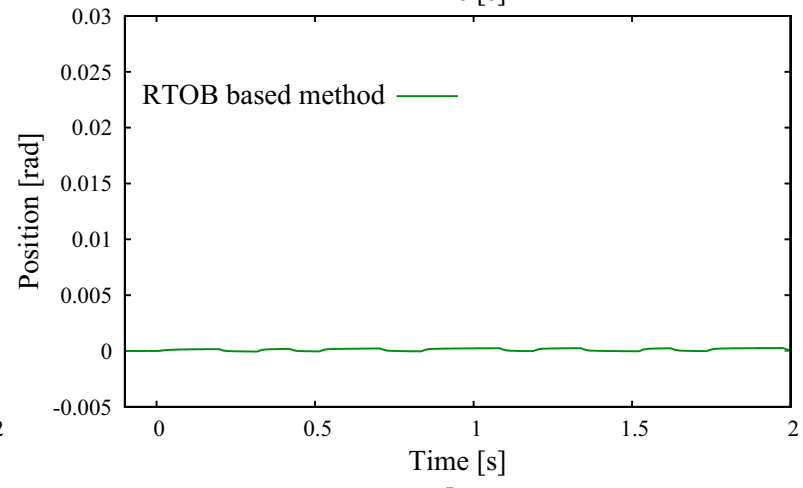

b
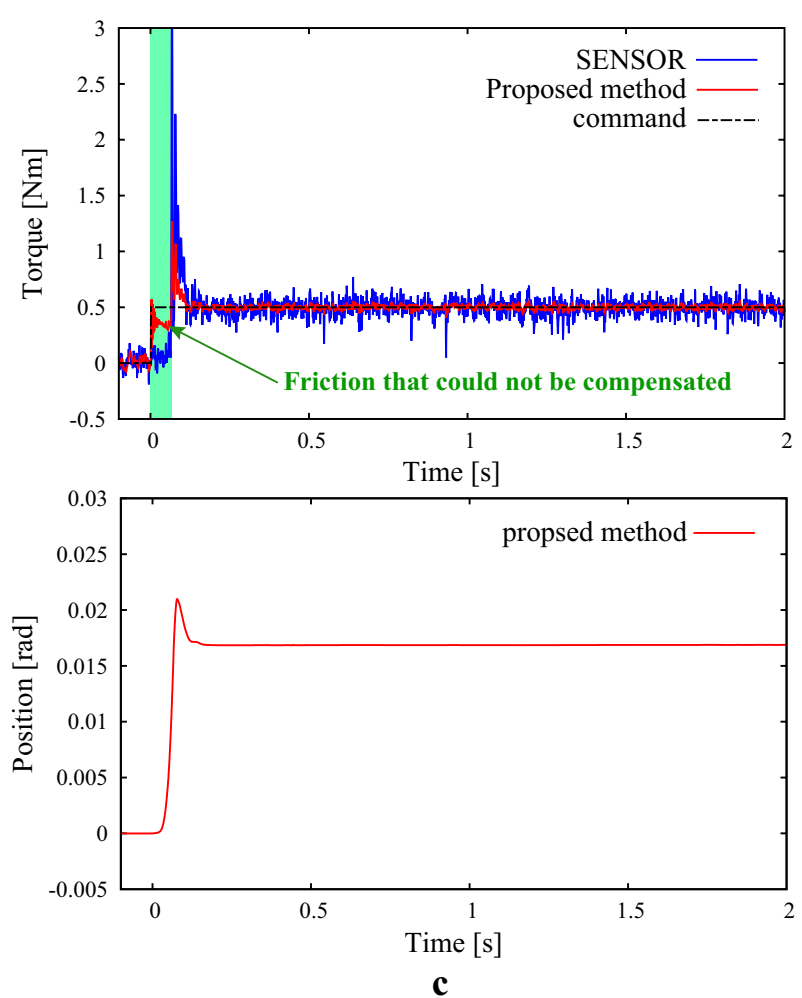

Fig. 16 Force and position responses of the force control systems. a Force control based on force sensor. b Force control based on RTOB. c Force control based on the proposed method 

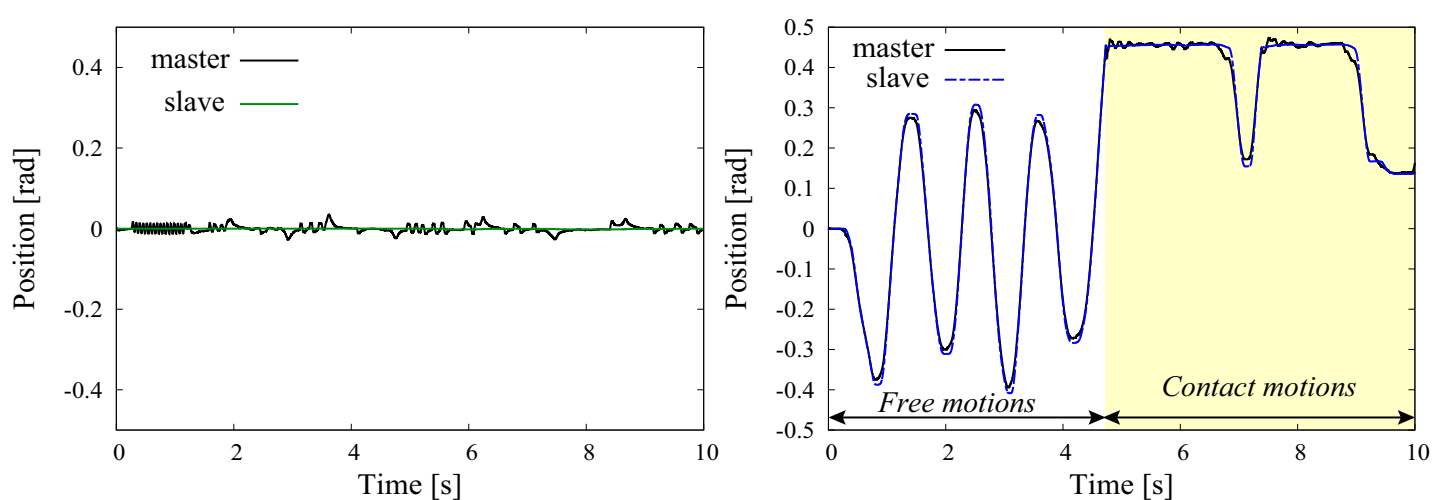

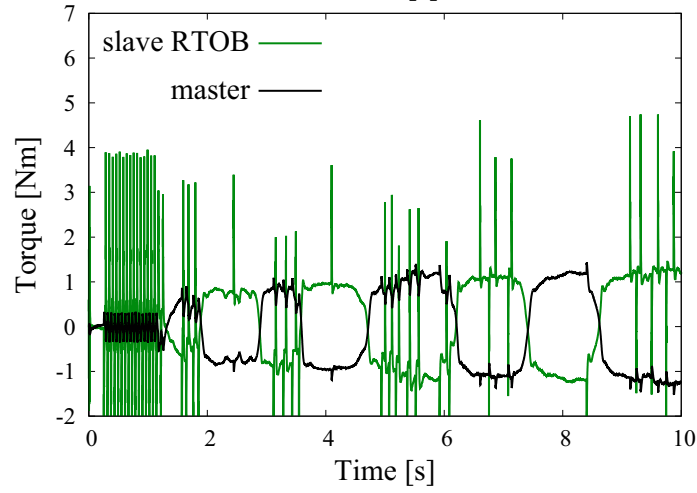

a

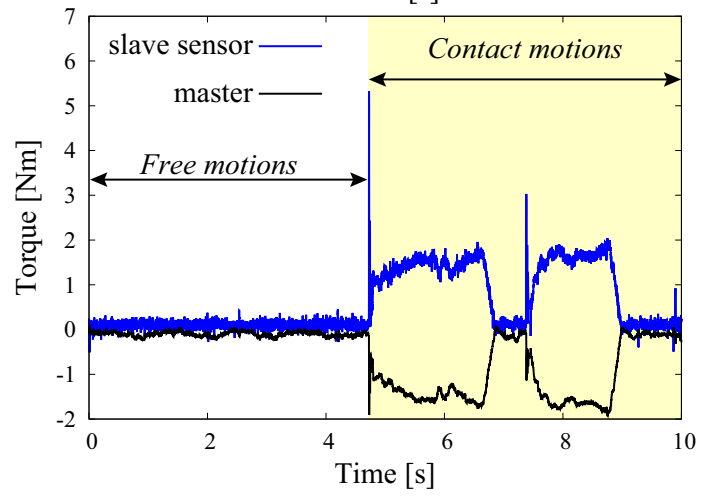

b

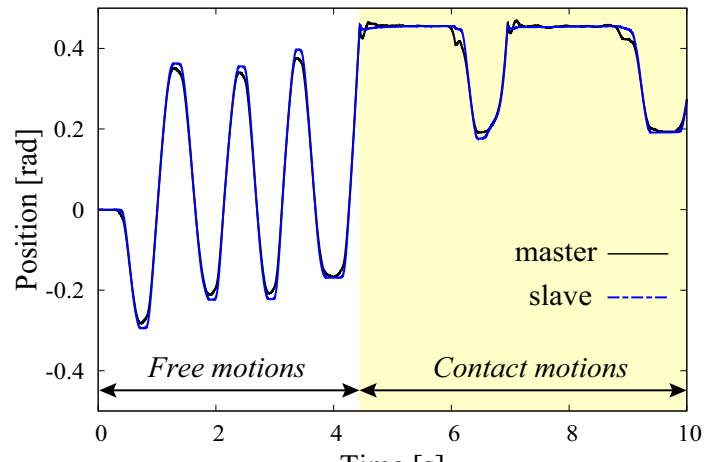

Time $[\mathrm{s}]$

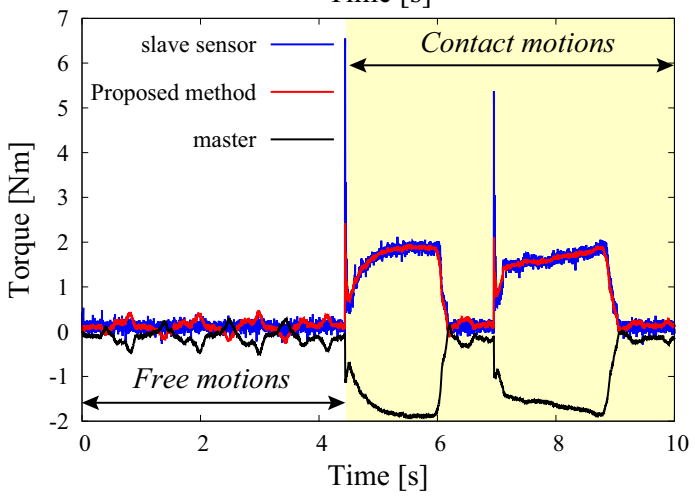

c

Fig. 17 Position and force responses of the bilateral control systems. a Bilateral control based on RTOB. b Bilateral control based on force sensor. $\mathbf{c}$ Bilateral control based on the proposed method 

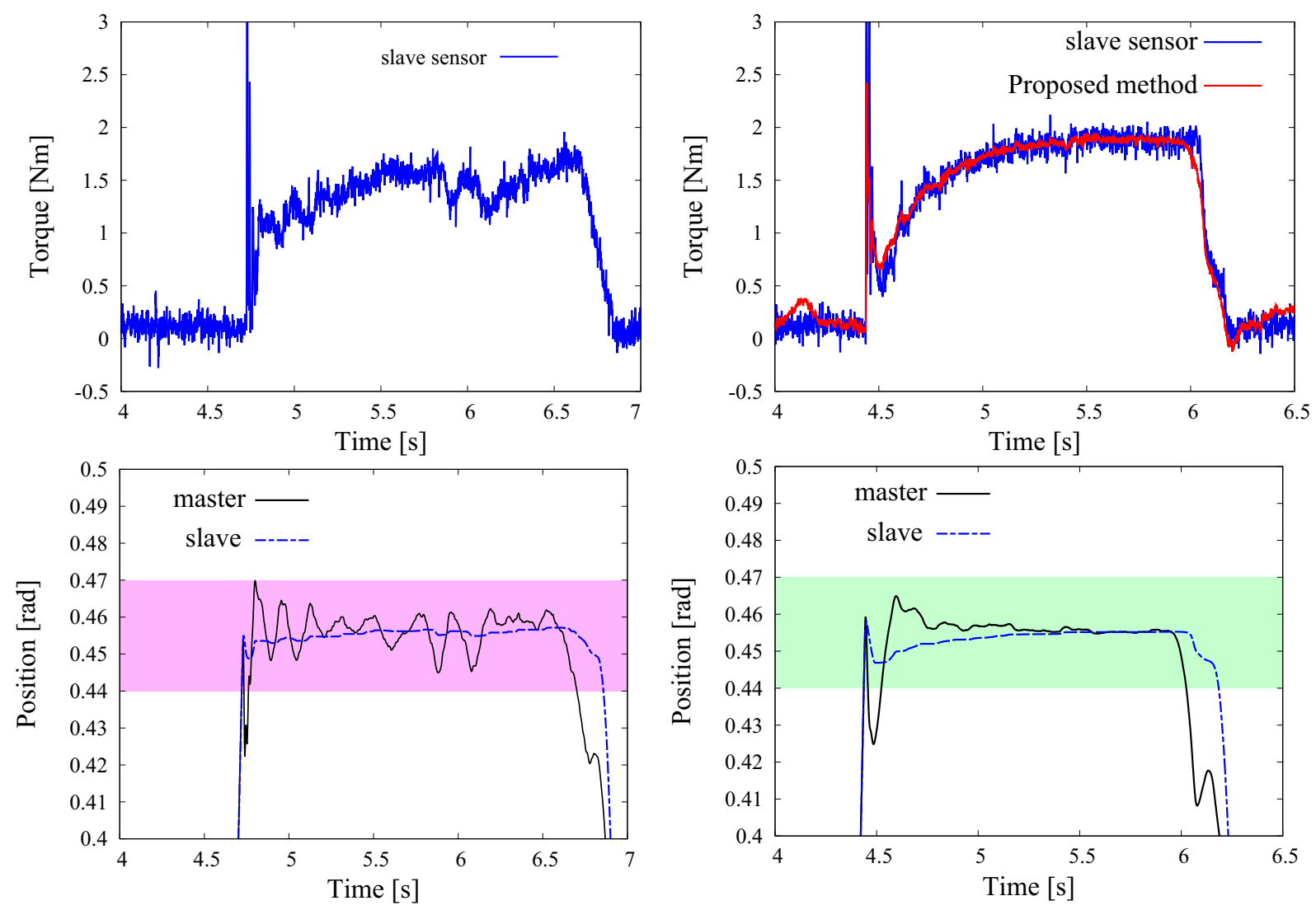

a

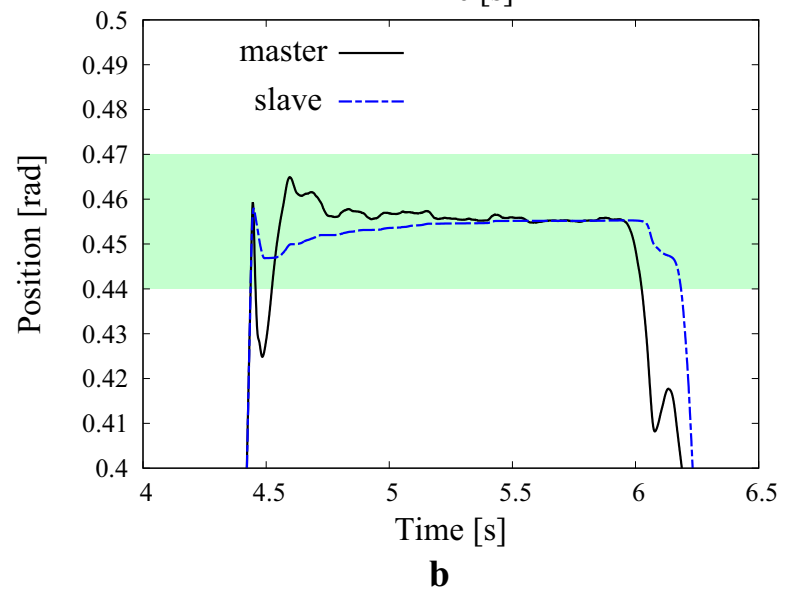

Fig. 18 Enlarged figures for position and force responses of the bilateral control systems. a Bilateral control based on force sensor. b Bilateral control based on the proposed method

Conversely, Fig. 18b shows that the proposed method achieves noise suppression and stable contact motions compared with the force sensor-based method.

The above results show the validity of the effectiveness of the proposed method.

\section{Conclusion}

This paper proposed a force control system by using an external-force estimation method based on the integration of RTOB and force sensor information. The conventional force control system by using a force sensor has difficulty in extending control bandwidth due to forcesensor noise. On the other hand, the RTOB-based force control system also has difficulty in the modeling, identification, and compensation of the friction torque (force), especially for the coulomb friction. Conversely, the proposed method achieves improving the friction torque (force) suppression performance and the noise sensitivity compared to the force-sensor based method and
RTOB-based method, respectively. The proposed method can improve the friction torque suppression performance by utilizing the low frequency-component of the force sensor. Additionally, the proposed method succeeds in improving the sensitivity to noise from the force sensor by using the high-frequency component of the estimated force by RTOB. The proposed external torque estimation method was applied to a force control system and a bilateral control system. The experimental results showed the validity of the proposed method.

As a future subject, the combination of the proposed estimation method with the Kalman filtering technology should be considered to achieve further improvement of the noise sensitivity in the case with the relatively lowresolution encoder. In addition, a detailed analysis of the relationship between the limitation of the integration bandwidth and the resolution of the position encoder should be addressed, though the integration bandwidth was determined by try and error in this paper. 


\section{Acknowledgements}

This work was supported in part by the Institute of Science and Engineering of Chuo University, and the Japan Society for the Promotion of Science under Grants-in-Aid for Scientific Research (KAKENHI) Grant Number 18K13780.

\section{Authors' contributions}

YN performed the analysis and experiments and drafted the manuscript. $\mathrm{HH}$ discussed the analysis and experiments with $\mathrm{YN}$ and helped to draft the manuscript. All authors read and approved the final manuscript.

\section{Funding}

Not applicable.

\section{Availability of data and materials}

The data that support the findings of this study are available from the corresponding author upon reasonable request.

\section{Competing interests}

The authors declare that they have no competing interests.

Received: 30 September 2019 Accepted: 5 February 2020

Published online: 17 February 2020

\section{References}

1. Nakamura T, Yamamoto A (2017) Modeling and control of electroadhesion force in dc voltage. Robomech J 4(1):18

2. Xu Q (2015) Robust impedance control of a compliant microgripper for high-speed position/force regulation. IEEE Trans Ind Electron 62(2):12011209. https://doi.org/10.1109/TIE.2014.2352605

3. Huard B, Grossard M, Moreau S, Poinot T (2015) Sensorless force/position control of a single-acting actuator applied to compliant object interaction. IEEE Trans Ind Electron 62(6):3651-3661. https://doi.org/10.1109/ TIE.2014.2367465

4. Ohishi K, Ohnishi K, Miyachi K (1983) Torque-speed regulation of DC motor based on load torque estimation method. Inst. of Electrical Engineers of Japan, Tokyo, pp 1209-1218

5. Ohnishi K, Shibata M, Murakami T (1996) Motion control for advanced mechatronics. IEEE/ASME Trans Mechatron 1(1):56-67. https://doi. org/10.1109/3516.491410

6. Sariyildiz E, Ohnishi K (2015) Stability and robustness of disturbanceobserver-based motion control systems. IEEE Trans Ind Electron 62(1):414-422. https://doi.org/10.1109/TIE.2014.2327009

7. Sariyildiz E, Ohnishi K (2015) On the explicit robust force control via disturbance observer. IEEE Trans Ind Electron 62(3):1581-1589. https:// doi.org/10.1109/TIE.2014.2361611

8. Murakami T, Yu F, Ohnishi K (1993) Torque sensorless control in multidegree-of-freedom manipulator. IEEE Trans Ind Electron 40(2):259-265. https://doi.org/10.1109/41.222648

9. Sariyildiz E, Ohnishi K (2015) An adaptive reaction force observer design. IEEE/ASME Trans Mechatron 20(2):750-760. https://doi.org/10.1109/ TMECH.2014.2321014

10. Fukushima Y, Naemura K (2014) Estimation of the friction force during the needle insertion using the disturbance observer and the recursive least square. ROBOMECH J 1(1):14

11. Katsura S, Matsumoto Y, Ohnishi K (2007) Modeling of force sensing and validation of disturbance observer for force control. IEEE Trans Ind Electron 54(1):530-538. https://doi.org/10.1109/TIE.2006.885459

12. Iida W, Ohnishi K (2004) Reproducibility and operationality in bilateral teleoperation. In: The 8th IEEE international workshop on advanced motion control, 2004. AMC '04., pp 217-222. https://doi.org/10.1109/ AMC.2004.1297669

13. Nagatsu Y, Katsura S (2015) Design strategies for motion reproduction based on environmental disturbance compensation. IEEE Trans Ind Electron 62(9):5786-5798. https://doi.org/10.1109/TIE.2015.2448062

14. Nagatsu Y, Katsura S (2017) Decoupling and performance enhancement of hybrid control for motion-copying system. IEEE Trans Ind Electron 64(1):420-431. https://doi.org/10.1109/TIE.2016.2596701

15. Jatta F, Legnani $G$, Visioli A (2006) Friction compensation in hybrid force/velocity control of industrial manipulators. IEEE Trans Ind Electron 53(2):604-613. https://doi.org/10.1109/TIE.2006.870682

16. Jamaludin Z, Van Brussel H, Swevers J (2009) Friction compensation of an $x y$ feed table using friction-model-based feedforward and an inversemodel-based disturbance observer. IEEE Trans Ind Electron 56(10):38483853. https://doi.org/10.1109/TIE.2009.2017560

17. Verbert KAJ, Tóth R, Babušaka R (2016) Adaptive friction compensation: a globally stable approach. IEEE/ASME Trans Mechatron 21(1):351-363. https://doi.org/10.1109/TMECH.2015.2428995

18. Shimoichi T, Katsura S (2013) Optimization method for disturbance compensation in bilateral control. IEEJ J Ind Appl 2(2):113-120. https:// doi.org/10.1541/ieejjia.2.113

19. Ruderman M, Iwasaki M (2015) Observer of nonlinear friction dynamics for motion control. IEEE Trans Ind Electron 62(9):5941-5949. https://doi. org/10.1109/TIE.2015.2435002

20. Maeda Y, Iwasaki M (2013) Initial friction compensation using rheologybased rolling friction model in fast and precise positioning. IEEE Trans Ind Electron 60(9):3865-3876. https://doi.org/10.1109/TIE.2012.2205350

21. Hayashida N, Yakoh T, Mmurakami T, Ohnishi K (2001) A sensorless bilateral robot manipulator based on twin drive system. J Jpn Soc Precis Eng 67(11):1834-1838. https://doi.org/10.2493/jjspe.67.1834

22. Komiyama H, Uchimura Y (2012) Contactless magnetic gear for robot control application. IEEJ Trans Ind Appl 132(3):389-396. https://doi. org/10.1541/ieejias.132.389

23. Komada S, Nomura K, Ishida M, Hori T (1993) Robust force control based on compensation for parameter variations of dynamic environment. IEEE Trans Ind Electron 40(1):89-95. https://doi.org/10.1109/41.184825

24. Murakami T, Ohnishi K (1992) Observer-based adaptive force control of multi-degrees-of-freedom manipulator. In: Proceedings of the 1992 international conference on industrial electronics, control, instrumentation, and automation, pp 1500-15053. https://doi.org/10.1109/IECON .1992 .254379

25. Park SS, Altintas Y (2004) Dynamic compensation of spindle integrated force sensors with kalman filter. J Dyn Syst Measur Control 126(3):443-452

26. Nam K, Oh S, Fujimoto H, Hori Y (2012) Estimation of sideslip and roll angles of electric vehicles using lateral tire force sensors through rls and kalman filter approaches. IEEE Trans Ind Electron 60(3):988-1000

27. Bätz G, Weber B, Scheint M, Wollherr D, Buss M (2013) Dynamic contact force/torque observer: sensor fusion for improved interaction control. Int J Robot Res 32(4):446-457. https://doi.org/10.1177/0278364913482015

28. Mitsantisuk C, Ohishi K, Urushihara S, Katsura S (2011) Kalman filter-based disturbance observer and its applications to sensorless force control. Adv Robot 25(3-4):335-353

29. Shimoichi T, Katsura S (2012) A method of friction identification based on optimization problem for real-world haptics. Trans Soc Instrum Control Eng 48(12):907-912. https://doi.org/10.9746/sicetr.48.907

\section{Publisher's Note}

Springer Nature remains neutral with regard to jurisdictional claims in published maps and institutional affiliations. 